Research Journal of Medical Sciences 6(1): 26-32, 2012

ISSN: $1815-9346$

(C) Medwell Journals, 2012

\title{
Blood Pressure Control Amongst Hypertensive Patients in a Tertiary Health Care Facility in Northern Nigeria
}

\author{
${ }^{1}$ Igbiks Tamuno and ${ }^{2}$ Musa Babashani \\ ${ }^{1}$ Department of Pharmacology, ${ }^{2}$ Department of Medicine, \\ Faculty of Medicine, Bayero University, Kano, Nigeria
}

\begin{abstract}
This study assessed the pattern of presentation of hypertensive patients in the clinic at Aminu Kano Teaching Hospital (AKTH) Kano, North western Nigeria, determined the level of control of hypertension in these patients and the drugs used for this purpose. A cross sectional retrospective study was conducted using case notes of 200 randomly selected patients who attended the hypertension clinic of the AKTH, Kano. Data extracted included pattern of presentation, drugs prescribed, adherence to treatment and control of blood pressure among the population studied including diabetics in the cohort. The study population had a mean age of 50.7 with over $30 \%$ being over 60 years. The $60 \%$ were females. Diabetes mellitus was the most common comorbid ailment $(13 \%)$ in the study population. Over 42 and $29.5 \%$ of the population presented with grade 3 and grade 2 hypertension, respectively. Over $80 \%$ of the patients were on combination therapy with over $42 \%$ of patients on at least 3 antihypertensive drugs. Diuretics were the most commonly prescribed drug group (34\%) with the vasodilator, hydrallazine, the least prescribed. About $53 \%$ of the patients were reported to have been regular on their drugs. Blood pressure was fully controlled in $34.5 \%$ of all patients and in $23.1 \%$ of diabetic patients in the study population. Control of blood pressure in the study population including diabetics was low while a high level of non adherence and low socio-economic status was seen in this study population calling for the use of multiple approaches including treatment intensification, scaling up of patient and provider education and creative health system policy changes to address the problem.
\end{abstract}

Key words: Hypertension, diabetes mellitus, adherence, blood pressure, AKTH, Nigeria

\section{INTRODUCTION}

Essential hypertension remains the most common cardiovascular disease in black Africans (Ajayi and Akinwusi, 1993) and a leading public health risk factor on a global scale. Hypertension has a higher age adjusted prevalence in Africa (Seedat, 2000) and its ravage is particularly severe. It is the leading cause of congestive heart failure, chronic renal failure, cerebrovascular disease, cardiovascular mortality and sudden cardiac death (Obasahan and Ajuyah, 1996; Rotimi et al., 1998; Adigun et al., 2003).

In a study of cardiovascular diseases in multiple centers in Nigeria, hypertension ranked first and its complications constituted $25 \%$ of emergency medical admissions in urban hospitals in the country (Ekere et al., 2005). A 1997 report by the International Collaborative study of hypertension in blacks put the age adjusted prevalence of hypertension in Nigeria at $14.5 \%$ (Cooper et al., 1997). However, using the current definition of hypertension from the seventh joint National Committee on Prevention, Detection, Evaluation and
Treatment of High Blood Pressure (JNCVII) guidelines (Anonymous, 2003), 20-25\% of Nigerians would be classified as hypertensives (Ogah, 2006). It is the medical illness most frequently diagnosed in the elderly (Bella et al., 1993) and is the most common condition associated with dementia in Nigeria. It is also the most common condition in senior executives and army recruits in Nigeria (Okojie et al., 2000; Awoyemi et al., 2001).

Treatment of hypertension has contributed to decrease in morbidity and mortality from cardiovascular diseases ( $\mathrm{Gu}$ et al., 2006). Individuals of lower socioeconomic status are known to have higher blood pressures and are more prone to cardiovascular disease (Kaplan and Keil, 1993). This in part reflects the higher prevalence of risk factors and the lower access to health care. One study in the United States found a direct correlation between subjects who have not had their blood pressure checked in the previous year and the socio-economic status (Schoenborn, 1998) while access to care may not totally explain the variation in mortality as seen in the various socio-economic classes reported, a 3 fold difference had been reported in coronary mortality

Corresponding Author: Igbiks Tamuno, Department of Pharmacology, Faculty of Medicine, Bayero University, Kano, Nigeria 
between the lowest and highest grades of employment with conventional risk factors only accounting for a modest proportion of the difference (Marmot et al., 1991). Cardiovascular mortality has also been associated with levels of education income, occupation and unemployment, poverty status and standard of living (Anonymous, 1997).

Uncontrolled hypertension remains a major threat to global health. The optimal target blood pressure is defined by the United States Joint National Committee on Prevention, Detection, Evaluation and Treatment of High Blood Pressure (JNCVII) according to whether the patient also has diabetes or a nephropathy. In uncomplicated hypertension, the target level is $140 / 90 \mathrm{mmHg}$. In the case of diabetes or nephropathy, it is $130 / 80 \mathrm{mmHg}$. In all cases, diet and exercise changes are also necessary and it is essential that patients understand them in order to comply with them. Epidemiological surveys have revealed that blood pressure control is adequate (i.e., $<140 / 90 \mathrm{mmHg}$ ) in only a small percentage of the hypertensive population (Burt et al., 1995; Marques-Vidal and Tuomilehto, 1997). In developed countries, only $27 \%$ of hypertensives on treatment had adequate blood pressure control (Colhoun et al., 1998). The scenario is worse in developing countries (Lopez and Murray, 1996).

Non compliance and poor adherence to therapeutic plans have been recognized as very important factors responsible for poor blood pressure control (Salako et al., 2003; WHO and ISH, 1999). Socio-economic factors have been adduced as a responsible factor for non compliance especially in Sub-Saharan Africa. Amidst the changing socio-economic paradigm in the environment, periodic assessment of blood pressure control and its associated factors are necessary.

This study was designed to assess the pattern of presentation of patients with hypertension attending the Hypertension Clinic of the Aminu Kano Teaching Hospital, Kano, North-Western Nigeria; determine the level of adequacy of blood pressure control in those with uncomplicated hypertension as well as those with diabetes; evaluate the drug groups used in these patients as well as their level of adherence. We hope the results of this study will serve as a useful information for possible future health intervention to improve patients treatment outcomes in these diseases.

\section{MATERIALS AND METHODS}

This cross sectional retrospective study was conducted between 3rd April and 10th June, 2009. A random sample of 200 case notes of patients with hypertension attending the hypertension (Specialist) clinic of the Aminu Kano Teaching Hospital, a tertiary health care facility located in Kano, North-West Nigeria. Data were extracted from the case notes using pre-piloted data collection forms. The data extracted from the case notes included: age, gender, systolic and diastolic blood pressure readings at first clinic attendance as well as co-morbid ailments. Others included:

- Antihypertensive drugs prescribed

- Patients adherence to prescribed drugs as documented by physicians in case notes

- Number of times prescribed drugs were changed

- Most recent systolic and diastolic blood pressure readings

- Before commencement of the study, ethical clearance for the study was granted by the ethical committee of the Aminu Kano Teaching Hospital, Kano

The classification of hypertension used in this study was based on the United States (U.S) Anonymous (2003) guideline. Data analysis was done with SPSS 16 for descriptive statistics and $\chi^{2}$-test with $p<0.05$ considered significant.

\section{RESULTS AND DISCUSSION}

About 200 randomly selected case notes of hypertensive patients were studied consisting of those belonging to $120(60 \%)$ females and 80 males (40\%). The patients had a mean age of $50.7+13.7$ with a range of $18-90$ years. About $60(30.5 \%)$ of the patients were over 60 years.

Table 1 shows the socio demographic and co morbid conditions associated with hypertension in the population studied. Nearly $50 \%$ of the study population were housewives while $20 \%$ were engaged in business and $5.5 \%$ of them were unemployed. Diabetes mellitus (13\%) was the most common co morbid ailment found in the study population while $15(7.5 \%), 13(6.5 \%), 13(6.5)$ and $3.5 \%$ patients had hyperlipidaemia, renal disease, obesity and congestive cardiac failure, respectively. All these patients were on appropriate therapy for their co morbid medical diseases.

Over $42 \%$ of the study population had grade 3 hypertension, nearly $30 \%(29.5 \%)$ had grade 2 hypertension while $13(6.5 \%)$ of them had isolated systolic hypertension at presentation. In all grades of hypertension, women were more preponderant at presentation (Table 2).

Blood pressure control was achieved in about 69 (34.5\%) patients while this was not attained in $70(35 \%)$ of the patients. In about $88(44 \%)$ of patients, the systolic blood pressure alone was controlled while in $110(55 \%)$, 
Res. J. Med.Sci., 6 (1): 26-32, 2012

Table 1: Socio-demographic and comorbid ailment distribution of study population

\begin{tabular}{lcc}
\hline Characteristic & Frequency & Percentage \\
\hline Gender & & \\
Male & 80.0 & 40.0 \\
Female & 120.0 & 60.0 \\
Age & & \\
Mean & 50.7 & - \\
SD & 13.7 & - \\
Range & 72.0 & - \\
Minimum & 18.0 & - \\
Max & 90.0 & - \\
Patients $\geq 60$ years & 60.0 & 30.5 \\
Occupation & & \\
Housewife & 97.0 & 48.5 \\
Business & 40.0 & 20.0 \\
Retired civil servants & 10.0 & 5.0 \\
Civil servants & 32.0 & 16.0 \\
Students & 5.0 & 2.5 \\
Farmers & 3.0 & 1.5 \\
Widows & 2.0 & 1.0 \\
Unemployed & 11.0 & 5.5 \\
Co-morbid ailments & & \\
Diabetes mellitus & 26.0 & 13.0 \\
Hyperlipidemia & 15.0 & 7.5 \\
Renal disease & 13.0 & 6.5 \\
Obesity & 13.0 & 6.5 \\
Peptic ulcer disease & 8.0 & 4.0 \\
Strolic & 8.0 & 4.0 \\
Congestive cardiac failure & 7.0 & 3.5 \\
Others & 15.0 & 7.5 \\
\hline
\end{tabular}

Table 2: Blood pressure distribution (According to grades) of study population at presentation

\begin{tabular}{|c|c|c|c|c|c|}
\hline Grades of BP & SBP/DBP & $\begin{array}{l}\text { No. of } \\
\text { subjects }\end{array}$ & $\mathrm{M}$ & $\mathrm{F}$ & $\%$ \\
\hline Grade 1 hypertension & $140-159 / 90-99$ & 43 & 16 & 27 & 21.5 \\
\hline Grade 2 hypertension & $160-179 / 100-109$ & 59 & 23 & 36 & 29.5 \\
\hline Grade 3 hypertension & $\geq 180 / \geq 110$ & 85 & 33 & 52 & 42.5 \\
\hline Isolated systolic hypertension & $\geq 140 /<90$ & 13 & 6 & 7 & 6.5 \\
\hline
\end{tabular}

only the diastolic blood pressure was controlled. Statistically significant difference was found in the fully controlled group among males and females as well as among the fully uncontrolled group $(\mathrm{p}<0.05)$ with more females having uncontrolled blood pressures (Table 3).

Among 26 patients with co-existing diabetes mellitus $(\mathrm{n}=26), 6(23.1 \%)$ had their blood pressures fully controlled while $13(0.5 \%)$ had fully uncontrolled blood pressures. In $8(30.8 \%)$ patients among all patients with diabetes mellitus, only the systolic blood pressure was controlled while only the diastolic blood pressure was controlled in $42.3 \%$ of the patients in this sub group.

About $66.6 \%$ (4) of diabetic patients with full blood pressure control were males while 2 out of $6(33.3 \%)$ diabetic patients with full control of their blood pressure had grade 3 hypertension at baseline with 3 patients $(50 \%)$ on grade 2 at baseline. About $17(65.4 \%)$ out of the 26 patients with diabetes mellitus in the cohort had grade 3 hypertension at baseline with $6(23.1 \%)$ patients in the grade 2 category at baseline.
Table 3: Pattern of BP control among all subjects in study population and patients with diabetes with goal $\mathrm{BP}$

\begin{tabular}{|c|c|c|c|}
\hline Treatments & Male $(\%)$ & Female (\%) & Total $(\%)$ \\
\hline \multicolumn{4}{|c|}{ With goal at $<140 / 90 \mathrm{mmHg}$} \\
\hline Fully controlled & $34(42.5)$ & $35(29.2)$ & $69(34.5)$ \\
\hline Fully uncontrolled & $23(28.8)$ & $47(39.2)$ & $70(35.0)$ \\
\hline Only SBP controlled & $41(51.3)$ & $47(39.2)$ & $88(44.0)$ \\
\hline Only DBP controlled & $52(65.0)$ & $58(48.3)$ & $110(55.0)$ \\
\hline \multicolumn{4}{|l|}{ At $<130 / 80 \mathrm{mmHg}$} \\
\hline Fully controlled & $4(23.5)$ & $2(22.2)$ & $6(23.1)$ \\
\hline Fully uncontrolled & $9(52.9)$ & $4(44.4)$ & $13(50.0)$ \\
\hline Only SBP controlled & $5(29.4)$ & $3(33.3)$ & $8(30.8)$ \\
\hline Only DBP controlled & $7(41.2)$ & $4(44.4)$ & $11(42.3)$ \\
\hline
\end{tabular}

Table 4: Summary of drug use pattem

\begin{tabular}{lcc}
\hline Characteristics & Frequency & Percentage \\
\hline Patients on monotherapy & 17 & 8.5 \\
Patients on fixed dose combination & 15 & 7.5 \\
Patients on 2 drug combination & 85 & 42.5 \\
Patients on 3 drug combination & 61 & 30.5 \\
Patients on 4 drug combination & 34 & 17.0 \\
Patients on 5 drug combination & 2 & 1.0 \\
Patients on 6 drug combination & 1 & 0.5 \\
Patients on antidiabetic drugs & 26 & 13.0 \\
Patients on lipid lowering drugs & 19 & 9.5 \\
\hline
\end{tabular}

Table 4 shows the drug use pattern among the study population. Only $8.5 \%$ were on monotherapy and over $42 \%$ had at least 3 antihypertensive drugs prescribed with 1 and $0.5 \%$ receiving 5 and 6 drugs, respectively.

About $81(53.0 \%)$ out of 153 patients were reported by their doctors to have been regular on their antihypertensives. The clinicians did not report on the adherence status of 47 patients in the study population. Diuretics, mainly thiazides were prescribed in $176(34 \%)$ out of 518 antihypertensive drugs prescribed for all subjects. This was followed by angiotensin converting enzyme inhibitors $(23.9 \%)$ and calcium channel blockers $(22.8 \%)$ while centrally acting antihypertensive drugs, mainly alpha methyl dopa was prescribed as $6 \%$ of total antihypertensives and angiotensin receptor blockers were prescribed in $4.6 \%$ of cases. Fixed dose combination drugs were prescribed in $2.9 \%$ of patients while the vasodilator hydrallazine was prescribed for only one patient.

Majority of the study population were in the middle age with a strong female preponderance $(60 \%)$. This agrees with other reports that hypertension is more common among females in middle age bracket (Hussain et al., 1999). Some researchers have opined that it is a reflection of the male populations poor attitude to keeping clinic appointments (Odili et al., 2008). Socio demographic distribution of the study population indicates that a large proportion of the study population were unemployed (Either as full time house wives, unemployed singles or married, students or widows) thus placing them in the lower socio economic groups with a 
preponderance of hypertension and its complications (Schoenborn, 1998; Marmot et al., 1991; Anonymous, 1997).

Diabetes mellitus was seen as the commonest co-morbid ailment in the study population $(13 \%)$ followed by hyperlipidemia $(7.5 \%)$. This finding is in agreement with previously conducted studies in Nigeria (Odili et al., 2008; Etuk et al., 2008; Yusuf and Balogun, 2005). Diabetes mellitus have been recognized as the most common complication of hypertension among black populations (Lopes, 2002). There is thus the need for aggressive treatment (Intensification) to achieve sustained control of blood pressure in these patients.

Over $>70 \%$ of the study population presented with grade 2 hypertension or higher. This finding agrees with those of other reports (Odili et al., 2008; Yusuff and Balogun, 2005). This observation is very worrisome especially against the finding that over $88 \%$ of the patients with Diabetes mellitus in this cohort had at least a grade 2 category hypertension on presentation. The patients with these conditions have an obviously higher cardiovascular risk and would therefore require tighter control of their blood pressures possibly with the use of multiple drugs.

The overall rate of blood pressure control in this study was $34.5 \%$ while $35 \%$ were fully uncontrolled. A statististically significant proportion of males compared to females had fully controlled blood pressures $(\mathrm{p}<0.05)$. However, the blood pressure levels themselves portrayed women to be more responsive, possibly lending credence to the opinion that women are more likely to adhere to therapeutic plan (Degoulet et al., 1983). The control rate of $34.5 \%$ is close to findings by Salako and his group at Ibadan who had a rate of $36 \%$ (Salako et al., 2003) while other workers had reported 30.5\% (Odili et al., 2008) and 29\% (Etuk et al., 2008), respectively. These are consistent with global trends in blood pressure control among hypertensives (Marques-Vidal and Tuomilehto, 1997; Burt et al., 1995). The negative impact of inadequate blood pressure control on morbidity and mortality is well documented (Obasahan and Ajuyah, 1996; Rotimi et al., 1998; Adigun et al., 2003). This is more so in this environment where late presentation, low socio economic levels and poor adherence to medication add to the burden of complications (Oyewo et al., 1989; Kadiri and Olutade, 1991).

The blood pressure control rate among diabetics in this study was $23.1 \%$. This, though lower than that seen in the general hypertensive population is higher than that reported by workers in Cameroun (Simeon et al., 2007) and those in other parts of the world (Teitelbaum et al., 2005; Wong et al., 2009) where control rates of $10.2,15.7$ and $28 \%$, respectively were reported.
Adequate blood pressure control is of particular concern in patients with diabetes as hypertension increases morbidity and mortality associated with stroke and cardiovascular disease (Johnansen and Birkeland, 2003) as well as microvascular complications such as retinopathy and nephropathy (Haffner, 1998). Evidence suggests that good blood pressure control may be as important if not more important than blood glucose control in reduction of the cardiovascular complications (UK Prospective Diabetes Study Group, 1998).

In this study, over $80 \%$ of the patients were on two or more drugs. This is in line with the recommendations of the JNC VII guidelines. In most cases, majority of hypertensive subjects will require more than one drug treatment to achieve optimal blood pressure control (Salako et al., 2003). However, inspite of the high rate of use of combination therapy, BP control was still less than satisfactory. In order to improve BP control rates, treatment intensification which has been found independently to improve blood pressure control levels in the setting of clinical trials and routine clinical practice (Okonofua et al., 2006) can be adopted. Even in the midst of poor adherence to therapy as seen in this cohort, treatment intensification may be useful in improving control rates. This has been demonstrated by Adam and colleagues (Rose et al., 2009) who showed that while treatment intensification was clearly associated with improved BP control over time this effect was similar in size for patients with varying levels of adherence. This argument is most reasonable when we note that many antihypertensive drugs have long half lives and drugs with long half lives may have a degree of forgiveness when some doses are missed (Urquhart, 1998).

Over $53 \%$ of the patients on whom adherence was reported were said to have complied to medication instructions. This level of compliance, even in the midst of the disadvantage of self report as a measure of adherence (Yusuf and Balogun, 2005) is not satisfactory and may have contributed to the low control. A better adherence level may have improved the blood pressure control level in this study population. Several studies have identified factors that may be responsible for the inadequate blood pressure control among Nigerian Hypertensives and these include late presentation for treatment, delayed diagnosis and commencement of treatment inadequacy of pharmacological treatment, non adherence to prescribed regimen, low socio economic/ under privileged class and subsequent inability to afford cost of drugs prescribed and exposure to stress (Kadiri et al., 2000; Oyewo et al., 1989; Kadiri and Olutade, 1991). 
Diuretics (34\%) either alone or in combination were the most commonly prescribed drug groups. This finding agrees with that of some researchers (Adigun et al., 2003) while other reports had other drug groups as the most frequently prescribed (Odili et al., 2008; Yusuff and Balogun, 2005; Chou et al., 2004). The high usage rate of diuretics is consistent with the recommendations of the JNC 7 guidelines and the working group of the International Society on Hypertension in Blacks. In addition to cost advantage, the volume dependency of hypertension in blacks and the ALLHAT study recommendation made strong cases for the use of diuretics as first line drugs. The seminal research of Wassertheil-Smoller and others also found that monotherapy with diuretics was equal or superior to others in preventing cardiovascular disease complications of high BP in women (Wassertheil-Smoller et al., 2004). Most of the diuretics prescribed were of the thiazide family, particularly bendrofluazide. Nevertheless, it must be pointed out that evidence from reviews and clinical trial data have found an increased incidence of new onset diabetes among patients receiving thiazide diuretics through mechanisms that are not fully understood but may be related to the association of thiazide induced hypokalemia with increased blood glucose which may be reversed by treating the hypokalemia (Zillich et al., 2006), The use of ACE inhibitors and calcium channel blockers were also most appreciable.

There was a ready recourse to the use of multitherapy in this study. Over $80 \%$ of patients were on combination therapy. This is in agreement with the JNC VII guidelines which states that small doses of different classes of antihypertensive drugs is more beneficial than a high dose of one. This advice probably accounts for $60 \%$ of antihypertensive drug visits in the United States getting polytherapy of three or more classes of drugs (Ma et al., 2006). The use of fixed dose combination therapy is rather low at $2.9 \%$. Fixed dose combinations have been known to improve adherence and consequently improve $\mathrm{BP}$ control rates.

In this study, life style modifications were not documented. Dietary indiscretion, especially liberal use of salt has been reported to have affected BP control (Salako et al., 2003). On the whole, improvement in control rates in this environment would entail the use of multiple strategies including treatment intensification, close adherence to clinical guidelines, development of a hypertension registry and disseminating best practices from high performing centers to other groups. This practice has been reported by a group in San Francisco, California to have increased control rates from $44 \%$ in $2001-80 \%$ by 2009 (Greenland et al., 2010). In an environment such as ours, the creative use of a hypertension registry and the use of non physician based clinic visits to measure and treat hypertension may contribute significantly to turn the tide against this disease.

\section{CONCLUSION}

The percentage of hypertensive patients with optimal blood pressure control in this study is low. Control of BP in patients with Diabetes as a comorbid ailment is also low, though higher than those reported by some other workers. Non compliance and low socio economic status and late presentation existed in this study population and may have contributed to the poor BP control observed. Multiple approaches including patient and provider education, treatment intensification, use of hypertension registry and other public health approaches and possible health system policy changes are needed to address these issues.

\section{ACKNOWLEDGEMENTS}

The researchers acknowledge the assistance of the staff of the medical records unit and the medical students of the 2009/2010 pharmacology class who helped with the sorting of files and some aspects of data collection and the secretarial assistance of Mr. Solomon of the Office of the Dean of medicine. The suggestions and editorial assistance of Dr. Joseph Fadare and Dr. (Mrs) Salawu are also gratefully acknowledged.

\section{REFERENCES}

Adigun, A.Q., D.A. Ishola, A.O. Akintomide and A.A.L. Ajayi, 2003. Shifting trends in the pharmacologic treatment of hypertension in a Nigerian tertiary hospital: A real-world evaluation of the efficacy, safety and rationality and pharmacoeconomics of old and newer antihypertensive drugs. J. Hum. Hypertens., 17: $277-285$.

Ajayi, A.A. and P.O. Akinwusi, 1993. Spectrum of hypertensive heart disease in Nigerians: A crosssectional study of echocardiographic indices and their correlation to treadmill exercise capacity. J. Hypertens., 11: 99-102.

Anonymous, 1997. The sixth report of the joint national committee on prevention, detection, evaluation and treatment of high blood pressure. Arch. Int. Med., 157: 2413-2446.

Anonymous, 2003. The seventh report of the joint national committee on prevention, detection, evaluation and treatment of high blood pressure. J. Am. Med. Assco., 289: 2560-2572. 
Awoyemi, A.O., C.K. Osagbemi and V.A. Ogunleye, 2001. Medical examination findings among army recruits in Illorin. West Afr. J. Med., 20: 256-258.

Bella, A.F., O. Baiyewu, A. Bamigboye, J.D. Adeyemi, B.A. Ikuesan and R.O. Jegede, 1993. The pattern of medical illness in a community of elderly Nigerians. Cent. Afr. J. Med., 39: 112-116.

Burt, V.L., J.A. Cutler, M. Higgins, M.J. Horan, D. Labarthe, P. Whelton and E.J. Roccella, 1995. Trends in prevalence, awareness, treatment and control of hypertension in the adult US population: Data from the health examination surveys 1960-1991. Hypertension, 26: 60-69.

Chou, C.C., M.S. Lee, C.H. Ke and M.H. Chung, 2004. Prescription patterns of hypertension-national health insurance in Taiwan. J. Chin. Med. Assoc., 67: 123-130.

Colhoun, H.M., W. Dong and N.R. Poulter, 1998. Blood pressure screening, managements and control in England: Results from the health survey for England 1994. J. Hypertens., 16: 752-757.

Cooper, R., C. Rotimi, S. Ataman, D. McGee and B. Osotimehin et al., 1997. The prevalence of hypertension in seven populations of west African origin. Am. J. Public Health, 87: 160-168.

Degoulet, P., J. Menard and H.A. Va, 1983. Factors predicting attendance at clinic and blood pressure control in hypertensive patients. Brit. Med. J., 287: 88-29.

Ekere, A.U., B.E. Yellowe and S. Umune, 2005. Mortality patterns in the accident and emergency department of an urban hospital in Nigeria. Niger J. Clin. Pract., 8: $14-18$.

Etuk, E., S.A. Isezuo, A. Chika, J. Akuche and M. Ali, 2008. Prescription pattern of anti-hypertensive drugs in a tertiary health institution in Nigeria. Ann. Afr. Med., 7: 128-132.

Greenland, P., J.S. Alpert, G.A. Beller, E.J. Benjamin and M.J. Budoff et al., 2010. ACCF/AHA guidelines for assesment of cardiovascular risk in asymptomatic adults: Executive summary. J. Am. Coll. Cardiol., 56: 2182-2199.

Gu, Q., R. Paulose-Ram, C. Dillon and V. Burt, 2006. Antihypertensive medication use among US adults with hypertension. Circulation, 113: 213 -221.

Haffner, S.M., 1998. The importance of hyper glycemia in the non fasting state to the development of cardiovascular disease. Endocr. Rev., 19: 583-592.

Hussain, A.A, A.G. Blzubier and M.E. Ahmed, 1999. Target organ involvement in hypertensive patients in Eastern Sudan. J. Hum. Hypertens., 13: 9-12.

Johnansen, O.E. and K.I. Birkeland, 2003. Preventing macrovascular disease in patients with type 2 diabetes mellitus. Am. J. Cardio. Vasc. Drugs, 3: 283-297.
Kadiri, S. and B.O. Olutade, 1991. The clinical presentation of malignant hypertension in Nigeria. J. Hum. Hypertens., 5: 339-343.

Kadiri, S., B.O. Olutade and O. Osabamiro, 2000. Factors influencing the development of malignant hypertension in Nigeria. J. Human. Hypertens., 14: 171-174.

Kaplan, G.A. and J.E. Keil, 1993. Socio-economic factors and coronary heart disease: a review of the literature. Circulation, 88 : 1973 -1998.

Lopes, A.A., 2002. Hypertension in black people: Pathophysiology and therapeutic aspects. J. Hum. Hypertension, 16: 11-12.

Lopez, A.D. and C.J. Murray, 1996. The global burden of diseases: A comprehensive assessment of mortality and disability from diseases, injuries and risk factors in 1990 and projected to 2020. Global Burden of Disease and Injury Series, Vol 1. Harvard School of Public Health, Cambridge.

Ma, J., K. Lee and R.S. Stafford, 2006. Changes in antihypertensive prescribing during US outpatient visits for uncomplicated hypertension between 1993 and 2004. Hypertension, 48: 846-852.

Marmot, M.G., S.G. Davey, S. Stansfield, C. Patel and F. North et al., 1991. Health inequalities among British civil servants: The Whitehall II Study. Lancet, 337: 1387-1393.

Marques-Vidal, P., J. Tuomilehto, 1997. Hypertension awareness, treatment and control in the community: Is the rule of halves still valid?. J. Hum. Hypertens., 11: 213-220.

Obasahan, A.O. and C.O. Ajuyah, 1996. How common is heart failure due to systemic hypertension alone in hospitalized Nigerians?. J. Hum. Hypertens., 10: $801-804$.

Odili, V.U., E.K. Oghagbon N.A. Ugwa, U.M. Ochei, O.E. Aghomo, 2008. Adherence to international guidelines in the management of HT in a tertiary hospital in Nigeria. Tr. J. Pharm. Res., 7: 945 -952.

Ogah, O.S., 2006. Hypertension in sub-saharan African populations: The burden of hypertension in Nigeria. Ethn. Dis., 16: 765-765.

Okojie, O.H., E.C. Isah and E. Okoro, 2000. Assessment of health of senior executives in a developing country. Public Health, 114: 273 -275.

Okonofua, E.C., K.N. Simpson, A. Jesri, S.U. Rehman, V.I. Durkalski and B.M. Egan, 2006. Therapeutic inertia is an impediment to achieving the healthy people 2010 blood pressure control goals. Hypertension, 47: 345-351.

Oyewo, E.A., A.A. Ajayi and G.A. Ladipo, 1989. A therapeutic audit in the management of hypertension in Nigeria. East Afr. Med. J., 66: 458-467. 
Rose, A.J., D.R. Berlowitz, M. Manze, M.B. Omer and N.R. Kessin, 2009. Intensifying therapy for hypertension despite suboptimal adherence. Hypertension, 54: 524-529.

Rotimi, O., A.A. Ajyi and W.O. Odesanmi, 1998. Sudden unexpected deaths due to cardiac causes: A review of 50 autopsied series in Nigerian. Int. J. Cardiol., $63: 111-115$.

Salako. B.L., F.A. Ajose and E. Lawani, 2003. Blood pressure control in a population where antihypertensives are given free. East. Afr. Med. J., 80: 529-531.

Schoenborn, C.A., 1998. Health Promotion and Disease Prevention. NationalCenter for Health Statistics, Hyattsville. Md.

Seedat, Y.K., 2000. Hypertension in developing nations of sub-saharan Africa. J. Hum. Hypertens., 14: 739-747.

Simeon, P.C., A.P. Kengue, Y.M. Dehayem, N.L. Simo, J.C. Mbanya, 2007. Hypertension in people with diabetes in sub-saharan Africa: Revealing the hidden face of the iceberg. Diabetes Res. Clin Pract., 77: 293-299.

Teitelbaum, B.A., D.K. Roberts, J. Ecktund-Winters, D.D. Castells and C.C. Alexander, 2005. Blood pressure control in an African American sample with diabetes mellitus in an Urban eyes clinc. Optometry, 76: 653-656.

UK Prospective Diabetes Study Group, 1998. Tight blood pressure control and risk of macrovascular and microvascular complications in type 2 diabetes: UKPDS 38. Br. Med. J., 317: 703-713.
Urquhart, J., 1998. Pharmacodynamics of variable patient compliance: implication for pharmaceutical value. Adv. Drug Deliv. Rev., 33: 207-219.

WHO and ISH, 1999. Guidelines for the management of hypertension. J. Hypertens., 17: 151-183.

Wassertheil-Smoller, S., B. Psaty, P. Greenland, A. Oberman and T. Kotchen et al., 2004. Association between cardiovascular outcomes and antihypertensive drug treatment in older women. J. Am. Med. Assoc., 292: 2849-2859.

Wong, N., S.A. Wang, E. Lamoureux, T.Y. Wong, G. Tikellis, A. Harper and J.J.J. Wang, 2009. Blood pressure control and awareness among patients with diabetes and hypertension attending a tertiary opthalmic clinic. Diabet Med., 26: 34-39.

Yusuf, K.B. and O. Balogun, 2005. Physicians prescribing of anti-Hypertensive combinations in a tertiary care setting in South Western Nigeria. J. Pharm. Acent. Sci., 8: 235-242.

Yusuff, K,B. and O.B. Balogun, 2005. Pattern of drug utilization among hypertensives in a Nigerian teaching hospital. Pharmacoepidemiol. Drug, Saf., 14: 69-74.

Zillich, A.J., J. Garg, S. Basu, G.L. Bakris and B.L. Carter, 2006. Thiazide diuretics, potassium and the development of diabetes: A qualitative review. Hypertension, 48: 219-224. 